\title{
P 134 THE INSERTION OF LONG-TERM PERITONEAL DRAINS IN PATIENTS WITH RECURRENT MALIGNANT ASCITES
}

Anna Brown, Alexandra Drake, Matthew Gibson, Madhumita Bhattacharyya, Lisa Clarke, Maeve Mckeogh. Palliative Care, Royal Berkshire Hospital, Reading, UK

\subsection{6/bmjspcare-2014-000654.175}

Background Recurrent malignant ascites poses a significant burden to oncology patients approaching the final phase of life. This is, in part, due to recurrent hospital admissions for intermittent paracentesis. In March 2012, the National Institute for Clinical Excellence approved the insertion of indwelling, longterm, peritoneal drains for recurrent malignant ascites. Guidance concluded clinical efficacy, low complication rates, cost efficiency (estimated saving $£ 679$ per patient) and improvement in patient quality of life.

Aims The Royal Berkshire Hospital had no long-term drain provision. The intention was to implement a trust-wide protocol for the insertion of long-term peritoneal drains in patients with recurrent malignant ascites.

Methods A pilot group was established with members from Oncology, Palliative Care and Radiology. A protocol was developed, presented and accepted at Clinical Governance by all relevant departments. The protocol was instituted via the trust intranet. Education regarding the process and the practicalities of the drains was provided at departmental level. Data was collected on patient experience.

Results Since introduction, eight months ago, twelve long-term drains have been placed. Malignant sites include ovarian (5), endometrial (3), urological (2), breast (1) and colorectal (1). Average drain duration is 37.5 days (range 2-215 to date). Placement of long-term drains enabled two patients to consider hospice care and two patients to die at home in their preferred place of care. Seven patients remain at home with ongoing community management of their ascites. Complication rates have been low with no insertion complications. In one case, two drains had to be inserted due to pocketed ascites. A further case displayed localised cellulitis at the insertion site but was not associated with systemic illness.

Conclusion The implementation of a trust-wide protocol has allowed the insertion of twelve long-term drains with optimisation of patient's quality of life, maximised time out of hospital and an estimated trust saving of $£ 8,148$. 